define dialogue, but it does give us a rich network of characteristics to look for when trying to appreciate a dialogue or understand how a work is anti-dialogical. As such it is a suggestion for the reading of dialogues that seems appropriate to dialogue, both because it came from Speroni, a writer of dialogue and an apology that is itself labyrinthine, but also because it is a complex image in which one can get lost, not a straightforward definition.

To conclude we can now speculate about Pugliese's solution to the larger problem I set out at the beginning of this review - how to understand dialogue in a way that shows what is at stake. One of the central features of the dialogue is its ability to encompass errant talk, whether it be digressions or potentially erroneous views. It is this willingness to err that distinguishes the authentic dialogue, but with this willingness comes the loss of certainty which comes with the introduction of digressions, distractions, and errant views. The dialogue that does not err and tries to get to the point, while clearer and better at the presentation of a single coherent thesis, would not be dialogical. We can all think of unsuccessful dialogues like Plato's Laws, where the presentation of a single view overwhelms the dialogue rendering it a dialogue in name only. By contrast, the dialogues we find most pleasant are those where we cannot with certainty say what the author's position is and when we try to do so we find ourselves wandering through a maze of interpretations. The dialogue form encourages, through the juxtaposition of characters and ideas, an uncertainty that frustrates the straightforward interpretation forcing the reader into a labyrinth of considerations. Pugliese's work does not lead us out of this maze, but shows how we might appreciate it for what it is and trace the contours of the labyrinth.

\title{
GEOFFREY ROCKWELL
}

McMasler Universily

Jackson I. Cope. Secret Sharers in Italian Comedy: From Machiavelli to Goldoni. Durham and London: Duke University Press, 1996. Pp. x, 221.

Il nome di Jackson I. Cope è ben noto agli studiosi del teatro italiano del Rinascimento, che hanno già avuto modo di apprezzare la vivacità delle sue intuizioni e l'impegno delle sue ricerche in lavori quali The Theater and the Dream: From Metaphor to Form in Renaissance Drama (Baltimore and London: Johns Hopkins UP, 1973) e Dramaturgy and the Daemonic: Studies in Anti-Generic Theater from Ruzante to Grimaldi (Baltimore and London: Johns Hopkins UP, 1984). In questa nuova opera il critico analizza una serie di testi più o meno noti partendo da una angolatura particolare: mentre nella struttura della Commedia Nuova di Plauto si assiste a un conflitto tra generazioni che si conclude con una rinnovata armonia fra i vecchi e i giovani (una struttura che si riflette nei testi a stampa della Commedia dell'Arte e in numerose occasioni della Commedia Erudita), nel corso del Rinascimento emerge quasi un sotto-genere di questa formula, il quale, pur conservando le componenti fondamentali dell'altro, presenta la circostanza in cui, nell'apparente rappacificazione finale, alcuni personaggi restano all'oscuro di qualcosa che è noto invece ad altri e al pubblico stesso. In tale prospettiva l'analisi si orienta ad approfondire in quei testi 
"secrecy as form, as a formal mode of dealing with a radical discrepancy between simultaneous stories unfolding in a single action doubly understood: one is the harmonious closure of New Comedy, the other the unfinished contingent plot"; la connivenza del pubblico nel mantenere questa segretezza sanziona la mancanza di circolarità nel testo, che rimane aperto a soluzioni di sotterfugi e di inganni "more cynical than carnivalesque" (5).

I precedenti di tale situazione sono chiaramente identificabili nel genere della novella; per limitarci a considerare il caso del Boccaccio (il cui nome ricorre spesso infatti nelle pagine del Cope), nei racconti di infedeltà coniugale il marito molte volte rimane all'oscuro del tradimento della moglie, noto invece al narratore, agli ascoltatori e ai lettori, e in quelli che presentano delle 'beffe', chi ne rimane vittima non sempre scopre di esserlo stato (come in 8.3 , dove Calandrino conserva la convinzione di aver davvero trovato l'elitropia), o qualcuno che ne è comunque coinvolto non ne sarà mai consapevole (in 9.8 Filippo Argenti non viene a sapere di esser stato usato da Ciacco per vendicarsi di Biondello). L'analisi del Cope parte proprio dalle analogie fra la novella di Alatiel (2.7) e la Philogenia di Ugolino Pisani, per proseguire nel Veneto con le situazioni de Li sei contenti di Galeotto del Carretto, della Veniexiana, e della Betía del Ruzzante. Lo studioso, che afferma esplicitamente di aver organizzato questo volume seguendo la geografia e non la cronologia, evitata (e talvolta capovolta) con l'intento "to minimize our inherent tendency to seek that progression which leads from an origin in $A$ to a full realization at $Z$ " (13) - nonostante nel primo risvolto si legga invece che "Cope's close and original readings of both classic and lesser-known plays [...] follow this peculiar Italian, anti-Plautine paradigm through variations across three centuries to its masterful and complex culmination in Carlo Goldoni's villeggiatura trilogy") -, si sofferma quindi su Siena, con La Raffaella del Piccolomini, i drammi rusticali e le loro trasformazioni in alcuni testi emanati dalla Congrega dei Rozzi (che anticipa l'Accademia degli Intronati), Firenze, con L'assiuolo del Cecchi, Il frate del Grazzini, La Mandragola del Machiavelli e Non bisogna in amor correre a furia del Fagiuoli, Milano, con Il manco male, Il Barone di Birbanza e I consigli di Meneghino del Maggi, per concludere con la Venezia del Goldoni, del quale sono presi in considerazione Il frappatore, La bottega del caffè, la trilogia della Villeggiatura e Una delle ultine sere di Carnevale. Intorno a questi testi si muovono poi moltissimı riferimenti ad altri autori e ad altre opere, confermando da un lato l'estensione delle letture del Cope e la sua capacità di spaziare nella storia della commedia italiana, e offrendo dall'altro un panorama quanto mai estensivo della stessa.

I limiti di questa recensione non consentono di ripercorre tutte le fini analisi dello studioso che mantengono sempre viva l'attenzione del lettore, messo costantemente di fronte ad aspetti originali nell'interpretazione di testi a volte poco noti, a volte ben conosciuti per altri motivi. Ci limitiamo a segnalare in particolare le puntuali precisazioni sulle differenze fra novella boccacciana e commedia, per cui mentre la prima "is a history, a completed stage of some protagonist's affairs that has been told to or shared by a teller who is retelling it, as we may do in turn. [...] If there is more to come, that is another story", nella seconda "the auditor does not know the outcome - the event presented is not detachable from its indefinite movement into the future" (61; e si veda anche il paragrafo "Boccaccio and Cinquecento Drama" [72-74]); e, in generale, gli accurati approfondimenti delle sfaccettature del tema e i sottili richiami fra i testi delle varie commedie esaminate. 
Spiacciono tuttavia alcune inesattezze che sfuggono al Cope e che avrebbero potuto trovare facilmente rimedio. Così si legge varie volte che una tipica situazione 'plautina' presente nella commedia rinascimentale è quella in cui protagonisti sono un marito vecchio, una moglie giovane e un potenziale seduttore (ad esempio a proposito della Mandragola [6]). In realtà nessuno di questi tre personaggi risale alla commedia classica, ma piuttosto al Boccaccio e al mondo della novella; evidentemente la società romana dei tempi di Plauto attribuiva troppa importanza al matrimonio per ammettere e accettare in questo modo l'adulterio. Per Plauto l'ingannato non è mai un marito, sì invece un padre o un ruffiano, e il giovane innamorato non mira a sedurre una moglie, ma a far l'amore con una ragazza che gli piace; se viene riconosciuta per donna libera, finirà con lo sposarla, mentre se si tratta di donna non libera che un ruffiano destina a fare la cortigiana, finirà col diventarne l'amante. E per rimanere alla Mandragola, non è vero che Callimaco "has been disguised as an expendable beggar who will impregnate Lucrezia and immediately thereafter die" (6); fa invece parte proprio del 'segreto' di cui Nicia rimane all'oscuro e che è noto ad altri personaggi e al pubblico il fatto che, nell'estensione nel futuro della vicenda, il padre dei bambini che nasceranno sarà Callimaco stesso e non Nicia, il quale rimane con la convinzione che lo sarà lui.

Allo stesso modo, mentre si deve certo essere d'accordo con il Cope quando scrive che tutte da rivedere sono le relazioni fra le commedie del Rinascimento per quanto riguarda la conoscenza diretta fra gli autori e i rapporti fra gli ambienti in cui quelle commedie erano scritte e rappresentate, una semplice verifica della cronologia avrebbe potuto porre rimedio alla frase secondo cui a Ferrara nel 1502 per il matrimonio di Alfonso d'Este e Lucrezia Borgia il fiorentino Lorenzo Strozzi avrebbe incontrato, "among the host of attendant luminaries, Ariosto and Ruzante" (15); all'altezza di quell'anno il primo, allora ventottenne, non poteva certo essere considerato tale, dal momento che non aveva scritto ancora nessuna commedia né, a quanto sappiamo, posto mano al poema. Per non parlare del secondo, che nel 1502 aveva sei anni.

Spiacciono poi le inesattezze nelle traduzioni dall'italiano o dal dialetto dei testi riportati: come il brano della Pellegrina, "[...] soleva dire sospirando un nostro cappellano: ch'a lui toccava l'uffiziare la chiesa, e un altro ne godeva l'entrata", che diventa, non molto chiaramente, "our curate used to say with a sigh: it was his job to take care of the church, while other people had the pleasure of entering" (202), mentre è evidente in primo luogo non trattarsi del "curato", ma di "un cappellano", e l'entrata di cui si parla è il reddito ricavato dalla chiesa (che va, verisimilmente, al curato stesso o ad altro beneficiario). $\mathrm{O}$ a proposito di un personaggio della Betía, chiamato regolarmente "Barba Scati" (40 sgg.), il lettore non familiare con il dialetto del Ruzzante non viene mai informato che "barba" significa semplicemente "zio", e non è parte del nome del personaggio stesso. Spiacciono infine, al lettore non ignaro della lingua italiana, i numerosi errori della grafia, davvero troppi per poter essere considerati dei semplici refusi, come quelli di lettere dell' alfabeto ("Pozzo" per "Pozza" [213] etc.), a volte omesse ("Intronti" per "Intronati" [199] etc.), di divisione in sillabe alla fine della riga ("Ca-rretto" per "Car-retto" [30] etc.), di accenti ("sè" per "sé" [32] etc.), di posizione e di spazi circa l'uso dell' apostrofo ("fra l'ventisette" per "fra '1 ventisette" [58], " a'miei" per "a' miei" [82] etc.), e via dicendo.

Nel complesso si deve comunque riconoscere che queste imperfezioni non sono tali da inficiare il sostanziale valore del volume e la validità delle ricerche del Cope, che 
mettono a fuoco una situazione assai interessante della storia della commedia italiana nel corso di tre secoli. Forse la tesi stessa richiede ulteriori chiarificazioni e approfondimenti, per lo meno nel senso che non sempre è facile distinguere, nei testi presi in considerazione, quale parte vi abbia l'esplicita volontà di ogni autore di adottare una struttura intenzionalmente antiplautina, e quanto invece sia la trama stessa a imporre una simile struttura, che riflette, come abbiamo visto, quella di gran parte delle novelle di beffa e di adulterio assente nel teatro classico. Ma questo studio apre indubbiamente delle nuove prospettive; il lavoro del Cope rimarrà di importanza centrale per ogni ricerca futura sul teatro comico italiano, e potrà insieme servire di modello per analoghe esplorazioni in altri campi e in altre letterature.

\section{ANTONIO FRANCESCHETTI \\ University of Toronto}

Giovanni Meli. Moral Fables and Other Poems. A Bilingual Anthology (Sicilian/English). Edited, introduced and translated into English Verse by Gaetano Cipolla. With Illustrations by Diane Miller, William Ronalds and Giuseppe Vesco. Brooklyn-Ottawa-Toronto: LEGAS, 1995. Pp. xxxix, 215.

Il Cipolla, già affermato traduttore del Meli - ha portato in versi inglesi L'origini di lu munnu (1985), Don Chisciotti e Sanciu Panza (1986) e Favuli morali (1988) - ripropone, in questa sua missione di far conoscere il massimo poeta in siciliano al pubblico di lingua inglese, un'antologia meliana in cui incorpora una versione rivista delle Origini di lu munnu e delle Favuli morali, nonché il canto 5 del Don Chisciotti e Sanciu Panza e altre poesie varie. Una di queste, il ditirambo "Sarudda", viene presentata per la prima volta in lingua inglese. L'intero volume è preceduto da un'ampia e pregevolissima introduzione dello stesso Cipolla, mirante ad inquadrare il Meli e le sue opere nell'ambiente culturale del Settecento. Le opere sono tutte corredate da numerose illustrazioni di Diane Miller (L'origini di lu munnu), William Ronalds (Favuli morali) e Giuseppe Vesco (Don Chisciotti e Sanciu Panza) che colgono nel segno lo spirito delle opere dell'abate palermitano ed aggiungono eleganza alla presente edizione.

L'introduzione - ampliata ed alquanto modificata rispetto alla versione apparsa nell'edizione delle Moral fables (Ottawa: Biblioteca di Quaderni d'italianistica, 1988) - è suddivisa in due parti. La prima presenta l'ambiente culturale, politico e sociale del '700 siciliano in cui visse il Meli. Questo ambiente non può essere dissociato dal flusso delle idee filosofiche nel resto dell'Europa e, scrive il Cipolla, è completamente errato assegnare al Meli l'etichetta di "poeta siciliano", se con essa si vuole identificare un individuo "whose intellectual concerns were entirely of regional or provincial nature" (xiii). Il mondo spirituale del Meli "cannot be comprehended unless it is framed against the background of the history of the ideas in Europe" (xiii). In questa sua analisi il Cipolla si riallaccia alla cosiddetta "polemica gentiliana" e prende una netta e ben precisa posizione al riguardo, affermando che "research has proven conclusively that Sicily, especially during the second half of the 18th century, was not the backward island completely cut off from civilization that thinkers such as Giovanni Gentile had supposed it was" (xiii). La figura del Meli che viene fuori da 